Somnologie $2023 \cdot 27: 45-50$

https://doi.org/10.1007/s11818-021-00327-3

Eingegangen: 13. Juni 2021

Angenommen: 6. August 2021

Online publiziert: 5 . Oktober 2021

(c) Der/die Autor(en) 2021

\section{Subtypen der Insomnie - exemplarische Ansätze und offene Fragen}

\author{
Kai Spiegelhalder · Fee Benz · Bernd Feige · Dieter Riemann \\ Klinik für Psychiatrie und Psychotherapie, Universitätsklinikum Freiburg, Medizinische Fakultät, Albert- \\ Ludwigs-Universität Freiburg, Freiburg, Deutschland
}

\title{
Zusammenfassung
}

Verschiedene Autorinnen und Autoren nehmen an, dass es klinisch nützlich und wissenschaftlich erkenntnisbringend sein könnte, Subtypen der Insomnie zu identifizieren, um diese spezifisch und damit möglicherweise effektiver zu behandeln, als dies derzeit geschieht. Im vorliegenden Beitrag werden folgende exemplarisch ausgewählte Ansätze zur Einteilung der Insomnie in Subtypen vorgestellt: 1) Einteilungen nach klinischen Symptomen; 2) Primäre vs. sekundäre Insomnie; 3) Subtypen nach ICSD-2; 4) Insomnie mit und ohne objektiv messbare kurze Schlafdauer; 5) Subtypen aus der Netherlands Sleep Registry. Anschließend werden die Stabilität der Zuordnung von einzelnen Patienten zu den verschiedenen Subtypen sowie die klinische Relevanz der Einteilungen kritisch diskutiert.

\section{Schlüsselwörter}

Ein- und Durchschlafstörungen · Klassifikation · Komorbidität · Behandlungserfolg · Schlafdauer

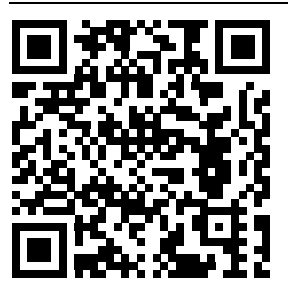

QR-Code scannen \& Beitrag online lesen

\section{Einleitung}

Wenn Patientinnen oder Patienten über Einschlafstörungen, Durchschlafstörungen oder frühmorgendliches Erwachen klagen und dies eine negative Auswirkung auf die Tagesbefindlichkeit oder Leistungsfähigkeit am Tag hat, kann die schlafmedizinische Diagnose Insomnie gestellt werden. Die diagnostischen Kriterien für die Insomnie erfüllen dabei sehr viele Menschen, in westlichen Industrienationen vermutlich etwa 5 bis $10 \%$ der gesamten Bevölkerung [21, 26].

In den letzten Jahren wurden weltweit mehrere Leitlinien veröffentlicht, in denen empfohlen wird, alle Erwachsenen mit einer Insomnie mittels der kognitiven Verhaltenstherapie für Insomnie (KVT-I) zu behandeln [23-25]. Schon sehr lange wird jedoch in der entsprechenden Literatur von verschiedenen Autorinnen und Autoren vorgeschlagen, dass es klinisch nützlich (und wissenschaftlich erkenntnisbringend) sein könnte, Subtypen der Insomnie zu identifizieren, um diese spezifisch und damit möglicherweise noch effekti- ver zu behandeln [7, 18, 27]. Im vorliegenden Beitrag werden folgende exemplarisch ausgewählte Ansätze zur Einteilung der Insomnie in Subtypen vorgestellt: 1) Einteilungen nach klinischen Symptomen; 2) Primäre vs. sekundäre Insomnie; 3) Subtypen nach ICSD-2; 4) Insomnie mit und ohne objektiv messbare kurze Schlafdauer; 5) Subtypen aus der Netherlands Sleep Registry. Anschließend werden verschiedene Probleme der Ansätze und offene Fragen diskutiert.

\section{Einteilungen nach klinischen Symptomen}

In aktuellen Klassifikationssystemen wird für die Diagnose Insomnie gefordert, dass eine Einschlafstörung, eine Durchschlafstörung oder ein frühmorgendliches Erwachen vorliegt. Dementsprechend unterscheiden verschiedene Autorinnen und Autoren zwischen den Subtypen „early insomnia“ (Einschlafstörung), "middle insomnia" (Durchschlafstörung) und "late insomnia" (frühmorgendliches Erwachen) [15]. Diese Einteilung hat mutmaßlich 
Tab. 1 Subtypen der Insomnie nach der zweiten Auflage der International Classification of Sleep Disorders (ICSD-2 [1])

Anpassungsbedingte (akute) Insomnie

Psychophysiologische Insomnie

Paradoxe Insomnie

Idiopathische Insomnie

Insomnie durch psychische Störung

Inadäquate Schlafhygiene

Verhaltensbedingte Insomnie in der Kindheit

Insomnie durch Medikation oder Substanzgebrauch

Insomnie durch körperliche Erkrankung

Nichtorganische Insomnie, nicht näher

bezeichnet

Organische Insomnie, nicht näher bezeichnet

Einfluss auf viele therapeutische Entscheidungen, z. B. im pharmakologischen Bereich, in dem viele Ärztinnen und Ärzte bei Durchschlafstörungen und frühmorgendlichem Erwachen Substanzen mit längeren Halbwertszeiten empfehlen als bei Einschlafstörungen. Darüber hinaus ist es möglich, dass bei Einschlafstörungen bzw. bei frühmorgendlichem Erwachen eine zirkadiane Komponente eine wichtige Rolle spielt [16], was ebenfalls einen Einfluss auf therapeutische Entscheidungen haben kann (z. B. durch zusätzliche Gabe von Melatonin oder durch die Empfehlung, Lichttherapie einzusetzen). Insgesamt ist die zeitliche Stabilität der Zuordnung individueller Betroffener zu diesen drei einzelnen Subtypen allerdings allenfalls moderat [22]. So finden sich auch im Lebensverlauf bei jüngeren Menschen eher Einschlafstörungen und bei älteren Menschen eher Durchschlafstörungen [20]. Eine weitere Einteilung, die auf den klinischen Symptomen basiert, ist die Unterscheidung in akute und chronische Insomnie, je nach Dauer der bestehenden Störung [12]. Diesbezüglich liegen erste klinische Studien vor, in denen eine verkürzte Version der KVT-I erfolgreich bei Patientinnen und Patienten mit akuter Insomnie (kürzer als drei Monate) eingesetzt wurde [13].

Interessanterweise ist uns keine Einteilung der Insomnie in Subtypen bekannt, die auf Unterschieden in der konkreten Ausprägung der Tagessymptomatik beruht, also beispielsweise auf der Un- terscheidung Konzentrationsstörung vs. Stimmungsbeeinträchtigung. Auch eine etablierte Einteilung nach dem Schweregrad der klinischen Symptome gibt es unseres Erachtens nicht, was möglicherweise einen Ansatz für zukünftige Entwicklungen bietet.

\section{Primäre vs. sekundäre Insomnie}

Ein- und Durchschlafstörungen treten sehr häufig gemeinsam mit anderen körperlichen und psychischen Erkrankungen auf [5, 9, 29]. Klinisch scheint dabei aus der Sicht vieler Ärztinnen und Ärzte in einigen Fällen eine kausale Beziehung zwischen den Erkrankungen vorzuliegen, üblicherweise derart, dass die körperliche oder psychische Erkrankung als direkte Ursache der Ein- und Durchschlafstörungen angesehen wird. Dies hat dazu geführt, dass in diagnostischen Klassifikationssystemen, beispielsweise in der vierten Auflage des Diagnostic and Statistical Manual of Mental Disorders (DSM-IV [3]) oder in der zweiten Auflage der International Classification of Sleep Disorders (ICSD-2 [1]) zwischen einer Insomnie ohne zugrundeliegende Grunderkrankung (primäre Insomnie) und einer Insomnie mit zugrundeliegender Grunderkrankung (sekundäre Insomnie) unterschieden wurde. Mit dieser Unterscheidung ging die Vorstellung einher, dass die primäre Insomnie mit einer spezifischen auf den Schlaf ausgerichteten Behandlung therapiert werden sollte, während bei der sekundären Insomnie eine Behandlung der Grunderkrankung angemessen und in vielen Fällen ausreichend sei. Somit hatten die Subtypen primäre und sekundäre Insomnie eine direkte Implikation für die klinische Behandlung der Patientinnen und Patienten.

Vor der Veröffentlichung des DSM-5 [4] wurde jedoch mehr und mehr deutlich, dass über die pathophysiologischen Mechanismen, die für den Zusammenhang zwischen Schlafstörungen und anderen Erkrankungen verantwortlich sind, sehr wenig bekannt ist. Zudem ist es klinisch äußerst schwierig zu entscheiden, ob im Einzelfall ein kausaler Zusammenhang vorliegt oder nicht. So bleibt zum Beispiel in vielen Fällen auch nach erfolgreicher Behandlung der vermeintlichen Grunderkrankung eine insomnische Residualsym- ptomatik bestehen. Darüber hinaus gibt es eine schnell zunehmende Menge an Daten, die deutlich machen, dass eine spezifische auf den Schlaf ausgerichtete Behandlung auch in solchen Fällen sehr hilfreich sein kann, in denen anderen Erkrankungen bei der gleichen Person vorliegen. So zeigte eine Metaanalyse für Patientinnen und Patienten mit komorbid vorliegender Insomnie eine Reduktion der subjektiven Einschlafzeit um $20 \mathrm{~min}$, eine Reduktion der subjektiven nächtlichen Wachzeit um 20 min, eine Erhöhung der subjektiven Schlafdauer um $17 \mathrm{~min}$ und eine Erhöhung der subjektiven Schlafeffizienz um 9\% nach Behandlung mit KVT-I [5]. In DSM-5 [4] und ICSD-3 [2] wurde die Unterscheidung in primäre und sekundäre Insomnie dementsprechend aufgegeben, und die Insomnie kann in diesen Klassifikationssystemen komorbid zu anderen Erkrankungen diagnostiziert und behandelt werden. Unseres Erachtens ist der Einfluss, den die Unterteilung in primäre und sekundäre Insomnien auf das Denken vieler ärztlicher Kolleginnen und Kollegen hatte, jedoch weiterhin deutlich erkennbar.

\section{Subtypen nach ICSD-2}

In der zweiten Auflage der International Classification of Sleep Disorders (ICSD-2 [1]) wurden 11 Subtypen der Insomnie unterschieden, die in $\mathbf{0}$ Tab. 1 dargestellt sind. Ähnlich wie bei der Unterscheidung in primäre und sekundäre Insomnie wurde diese Einteilung in 11 Subtypen der Insomnie jedoch auch mit der dritten Auflage der International Classification of Sleep Disorders (ICSD-3 [2]) wieder abgeschafft. Dabei dürfte eine wissenschaftliche Arbeit von Edinger et al. eine maßgebliche Rolle gespielt haben, in der sich die Reliabilität der ICSD-2-Subtypen im Vergleich verschiedener Beurteilerinnen und Beurteiler empirisch als relativ schwach herausgestellt hat [11]. Dies betraf insbesondere die Subtypen psychophysiologische (Interrater-Reliabilitäten von $0,12-0,55$ ) und paradoxe Insomnie (Interrater-Reliabilitäten von 0,12-0,51). Trotz der hohen Augenscheinvalidität gibt es unseres Wissens nach insgesamt auch nur wenige randomisierte klinische Studien, in denen Behandlungen spezifisch für einen der ICSD- 
2-Subtypen der Insomnie untersucht wurden.

\section{Insomnie mit und ohne objektiv messbare kurze Schlafdauer}

Vgontzas et al. schlugen im Jahr 2013 vor, die Insomnie in zwei Subtypen zu unterteilen, und zwar in die Insomnie mit und ohne objektiv messbare kurze Schlafdauer [27]. Die Insomnie mit objektiv messbarer kurzer Schlafdauer zeichne sich demnach durch eine genetische Prädisposition, physiologisch messbares Hyperarousal, eine objektiv messbare Verminderung der kognitiven Leistungsfähigkeit und durch ein erhöhtes Risiko für kardiovaskuläre Erkrankungen aus. Im Gegensatz dazu sei die Insomnie ohne objektiv messbare kurzeSchlafdauer durch erhöhte Ängstlichkeit und eine Neigung zum Grübeln gekennzeichnet. Diese Annahmen beruhten auf empirischen Studien der Forschungsgruppe von der Pennsylvania State University. Sie wurden überwiegend an einer Stichprobe von mehr als 1700 Versuchspersonen durchgeführt, wobei alle Versuchsper- sonen auch für eine Nacht polysomnografisch im Schlaflabor untersucht wurden. Vgontzas et al. [27] stellten die Hypothese auf, dass die Insomnie mit objektiv messbarer kurzer Schlafdauer eher pharmakologisch behandelt werden könne, wohingegen für die Insomnie ohne objektiv messbare kurze Schlafdauer eher eine psychotherapeutische Behandlung angemessen sei. Bislang liegt nur eine veröffentlichte prospektive klinische Studie zu dieser Thematik vor. Vgontzas et al. [28] wiesen dabei 15 Patientinnen und Patienten mit Insomnie mit objektiv messbarer kurzer Schlafdauer randomisiert einer psychotherapeutischen Behandlung mit KVT-I oder einer pharmakologischen Behandlung mit Trazodon zu. Auch wenn sich keine Unterschiede in der Wirksamkeit der Behandlung auf die Schwere der Insomnie zeigten, war die pharmakologische Behandlung in Bezug auf die objektiv messbare Schlafdauer und in Bezug auf eine Reduktion von Cortisol-Spiegeln der KVT-I überlegen. Publizierte Sekundäranalysen von klinischen Studien zeigen jedoch insgesamt gemischte Ergebnisse mit Studien, welche die Hy- pothese von Vgontzas et al. [27] unterstützen $[6,19]$, und anderen, welche dies nicht tun $[10,14,17]$. Zukünftige größere prospektive Studien werden diesbezüglich möglicherweise für Klarheit sorgen.

Unserer Auffassung nach ist es insgesamt eher ungünstig, dass die Zuordnung von Einzelpersonen zur Einteilung von Vgontzas et al. [27] im zeitlichen Verlauf relativ instabil ist. Bei den in - Abb. 1 dargestellten eigenen Daten aus dem Schlaflabor der Klinik für Psychiatrie und Psychotherapie des Universitätsklinikums Freiburg würden $15 \%$ (18 von 123) der Patientinnen und Patienten mit nichtorganischer Insomnie sowohl aufgrund der ersten als auch aufgrund der zweiten Nacht im Schlaflabor stabil dem Subtyp „Insomnie mit objektiv messbarer kurzer Schlafdauer" zugeordnet werden, und $50 \%$ (62 von 123) würden stabil dem Subtyp "Insomnie ohne objektiv messbare kurze Schlafdauer" zugeordnet werden. Allerdings würden auch $35 \%$ der Patientinnen und Patienten aufgrund der ersten Nacht im Schlaflabor einem anderen Subtyp zugeordnet werden als aufgrund

\section{Hier steht eine Anzeige.}




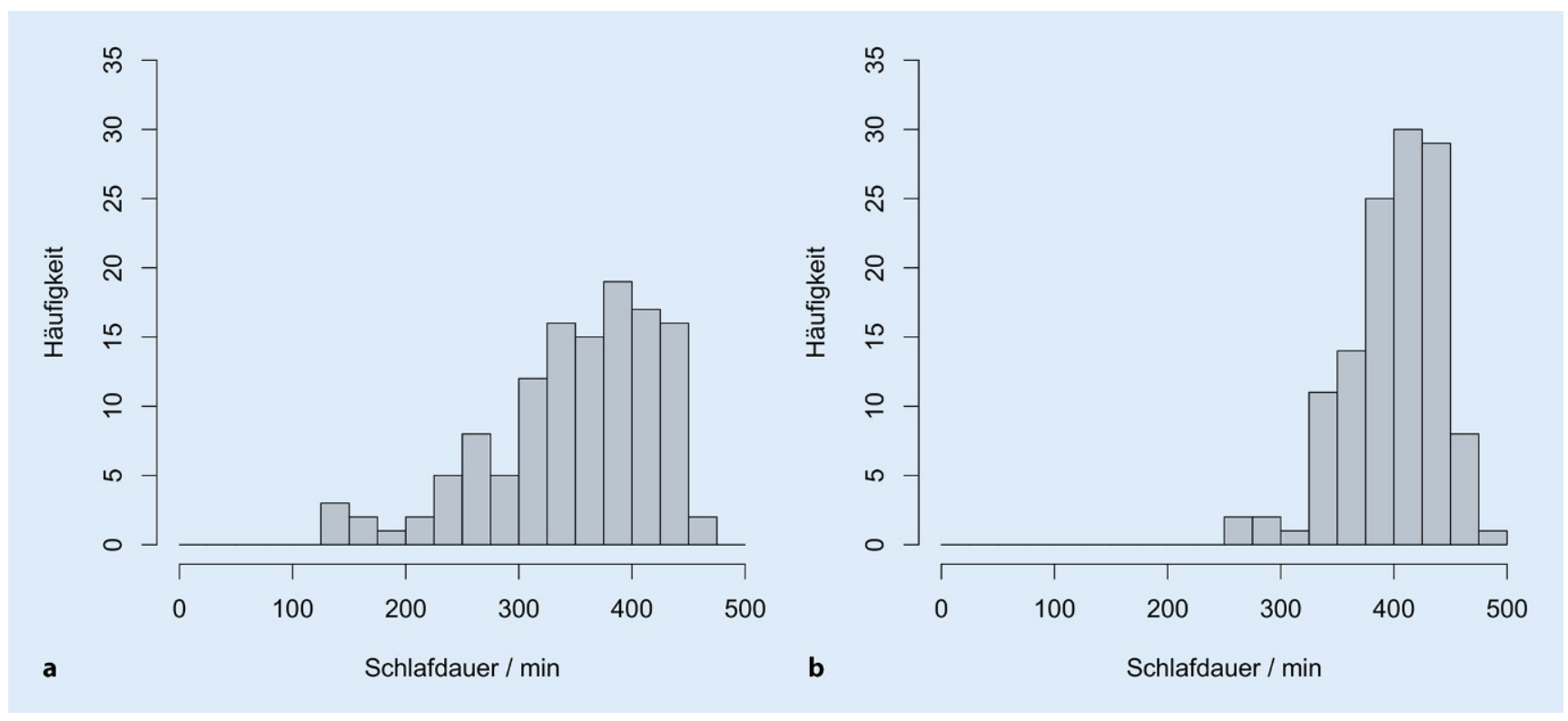

Abb. $1 \Delta$ Abbildung der Schlafdauer einer Stichprobe $(n=123)$ aus Patientinnen und Patienten mit Insomnie ohne komorbide körperliche oder psychische Erkrankung, die im Schlaflabor der Klinik für Psychiatrie und Psychotherapie des Universitätsklinikums Freiburg untersucht wurden. a Verteilung Schlafdauer, 1. Nacht; b Verteilung Schlafdauer, 2. Nacht

der zweiten Nacht im Schlaflabor. Insofern erscheint es fraglich, ob auf Basis einer festen Grenze für die Schlafdauer einer einzelnen Nacht im Schlaflabor weitreichende therapeutische Entscheidungen getroffen werden sollten. Formal gesehen müsste im Extremfall im Tagesrhythmus die Behandlung gewechselt werden, was selbstverständlich kein sinnvolles Vorgehen ist. Zudem ist die Interpretierbarkeit einzelner Schlaflabor-Nächte durch den so genannten "first night effect" eingeschränkt, und die polysomnografisch gemessene Schlafdauer bei Patientinnen und Patienten mit Insomnie weist üblicherweise keine bimodale Verteilung auf (- Abb. 1). Somit ist eine klare Trennung der beiden Gruppen kaum möglich.

\section{Subtypen aus der Netherlands Sleep Registry}

Der Ansatz einer Arbeitsgruppe um Eus van Someren aus Amsterdam basiert auf einer Analyse von Daten der sogenannten Netherlands Sleep Registry, einer onlinebasierten Erhebung von schlafbezogenen und allgemeinen gesundheitsbezogenen Variablen, Persönlichkeitseigenschaften und lebensgeschichtlichen Ereignissen bei mehr als 4000 Versuchspersonen aus den Niederlanden, von denen etwa die Hälfte einen Score von mindestens
10 Punkten im Insomnia Severity Index aufwies [7, 8]. Mit diesem datengetriebenen („evidenzbasierten") Ansatz möchte sich die Arbeitsgruppe dabei von vorherigen „eminenzbasierten“ Ansätzen abheben. Die Analyse der Daten, die auf der Methode der latenten Klassenanalyse beruhte, ergab fünf Subtypen der Insomnie, die als "sehr unglücklich", "moderat unglücklich, aber belohnungssensitiv“, "moderat unglücklich und nicht belohnungssensitiv“, ",wenig unglücklich mit hoher Reaktivität auf Lebensereignisse" und "wenig unglücklich mit niedriger Reaktivität auf Lebensereignisse" bezeichnet wurden [8]. Eine erneute Untersuchung etwa fünf Jahre später ergab, dass die Zuordnung zu den Subtypen relativ stabil war [8]. So betrug die Wahrscheinlichkeit, vor und nach diesen fünf Jahren dem gleichen Subtyp zugeordnet zu werden, $87 \%$.

Auch wenn der Ansatz einer datenbasierten Einteilung von Subtypen sicherlich sehr vielversprechend ist, muss angemerkt werden, dass die Auswahl der Messinstrumente selbstverständlich auch durch die niederländische Arbeitsgruppe und damit durch Expertinnen und Experten vorgenommen wurde. So ist es durch die Netherlands Sleep Registry beispielsweise auch nicht möglich, die Subtypen Insomnie mit und ohne objektiv messbare kurze Schlafdauer zu identifizieren, weil die Datenbank keine objektiven schlafbezogenen Maße enthält. Zudem liegen zu diesem relativ neuen Ansatz bislang keine Studien vor, in denen die Bedeutung der Subtypen für die Behandlung der Insomnie eingehend prospektiv untersucht wurde.

\section{Diskussion}

Im vorliegenden Beitrag wurden exemplarisch verschiedene Ansätze dargestellt, mit denen die Insomnie in Subtypen unterteilt werden kann. Dabei wurden sowohl ältere Ansätze eingeschlossen, die zum Teil Bestandteil diagnostischer Klassifikationssysteme waren, dann aber wieder entfernt wurden, als auch neuere Ansätze, die bislang nicht in diagnostische Klassifikationssysteme aufgenommen wurden.

Grundsätzlich erscheinen solche Einteilungen der Insomnie in Subtypen klinisch und wissenschaftlich hilfreicher zu sein, bei denen die Zuordnung von Personen zu den Subtypen eine gewisse zeitliche Stabilität aufweist. Dabei ist der auf der Netherlands Sleep Registry beruhende Ansatz positiv hervorzuheben, bei dem die zeitliche Stabilität über einen längeren Zeitraum untersucht wurde und sich auch als sehr hoch herausgestellt hat. Bei anderen Ansätzen, z.B. der Einteilung in Insomnie mit und ohne objektiv messbare kurze Schlafdauer, ist es hingegen relativ offenkundig, dass 
die zeitliche Stabilität der Einteilung eher gering ist.

Generell ist es unser subjektiver Eindruck, dass sich die neueren Ansätze (Insomnie mit und ohne objektiv messbare kurze Schlafdauer, Subtypen aus der Netherlands Sleep Registry) klinisch bislang kaum etablieren konnten. Dies hängt neben der Neuheit der Ansätze vermutlich auch damit zusammen, dass es bislang keine überzeugende Evidenz dafür gibt, dass diese Einteilungen eine klinische Relevanz für therapeutische Entscheidungen haben. Dies war vermutlich auch ein sehr wichtiger Grund dafür, dass frühere Einteilungen (primäre vs. sekundäre Insomnie, Subtypen nach ICSD-2) wieder aus den entsprechenden Klassifikationssystemen entfernt wurden. Dabei ist bemerkenswert, dass es unseres Wissens nach insgesamt nur sehr wenige randomisierte klinische Studien gibt, in denen spezifisch die Behandlung von definierten Subtypen der Insomnie untersucht wurde. So ergibt beispielsweise die Suche nach "(paradoxical insomnia) AND randomized" einen Treffer und die Suche nach "(idiopathic insomnia) AND randomized" drei Treffer in PubMed, wobei sich nur einer dieser Treffer auf eine randomisierte klinische Studie bezieht. Zudem werden unserer Erfahrung nach in vielen klinischen Studien nicht alle Informationen berichtet, die erforderlich sind, um einzuschätzen, wie stark welche Subtypen in einer Stichprobe vertreten sind. Diesbezüglich wäre unseres Erachtens eine Verbesserung des Erkenntnisstands im nächsten Jahrzehnt sehr zu begrüßen.

Korrespondenzadresse

\section{Prof. Dr. Dr. Kai Spiegelhalder}

Klinik für Psychiatrie und Psychotherapie, Universitätsklinikum Freiburg, Medizinische Fakultät, Albert-Ludwigs-Universität Freiburg Hauptstr. 5, 79104 Freiburg, Deutschland kai.spiegelhalder@uniklinik-freiburg.de

Funding. Open Access funding enabled and organized by Projekt DEAL.

\section{Einhaltung ethischer Richtlinien}

Interessenkonflikt. K. Spiegelhalder, F. Benz, B. Feige und D. Riemann geben an, dass kein Interessenkonflikt besteht.

\section{Insomnia subtypes-exemplary approaches and open questions}

Different authors have suggested that it may be clinically useful and scientifically valuable to identify robust subtypes of insomnia to increase treatment efficacy. Five exemplary classifications of insomnia subtypes are described in the current narrative review: 1) classifications according to clinical symptoms; 2 ) primary vs. secondary insomnia; 3) ICSD-2 subtypes; 4) insomnia with objective short or normal sleep duration; and 5) subtypes from the Netherlands Sleep Registry. Subsequently, temporal stability of subtype membership as well as clinical relevance of subtype classifications are critically discussed.

\section{Keywords}

Sleep initiation and maintenance disorders - Classification - Comorbidity - Treatment outcome . Sleep duration

Für diesen Beitrag wurden von den Autoren keine Studien an Menschen oder Tieren durchgeführt. Für die aufgeführten Studien gelten die jeweils dort angegebenen ethischen Richtlinien.

Open Access. Dieser Artikel wird unter der Creative Commons Namensnennung 4.0 International Lizenz veröffentlicht, welche die Nutzung, Vervielfältigung, Bearbeitung, Verbreitung und Wiedergabe in jeglichem Medium und Format erlaubt, sofern Sie den/die ursprünglichen Autor(en) und die Quelle ordnungsgemäßnennen, einen Link zur Creative Commons Lizenz beifügen und angeben, ob Änderungen vorgenommen wurden.

Die in diesem Artikel enthaltenen Bilder und sonstiges Drittmaterial unterliegen ebenfalls der genannten Creative Commons Lizenz, sofern sich aus der Abbildungslegende nichts anderes ergibt. Sofern das betreffende Material nicht unter der genannten Creative Commons Lizenz steht und die betreffende Handlung nicht nach gesetzlichen Vorschriften erlaubt ist, ist für die oben aufgeführten Weiterverwendungen des Materials die Einwilligung des jeweiligen Rechteinhabers einzuholen.

Weitere Details zur Lizenz entnehmen Sie bitte der Lizenzinformation auf http://creativecommons.org/ licenses/by/4.0/deed.de.

\section{Literatur}

1. American Academy of Sleep Medicine, AASM (2005) International classification of sleep disorders: diagnostic and coding manual, 2. Aufl. AASM, Westchester

2. American Academy of Sleep Medicine (2014) The international classification of sleep disorders, 3. Aufl. AASM, Darien

3. American Psychiatric Association, APA (1994)DSMIV: diagnostic and statistical manual of mental disorders, 4. Aufl. APA, Washington

4. American Psychiatric Association, APA (2013) DSM5: diagnostic and statistical manual of mental disorders, 5. Aufl. APA, Airlington

5. Baglioni C, Nanovska S, Regen W, Spiegelhalder K, Feige B, Nissen C, Reynolds CF, Riemann D (2016) Sleep and mental disorders: a metaanalysis of polysomnographic research. Psychol Bull 142:969-990

6. Bathgate CJ, Edinger JD, Krystal AD (2017) Insomnia patients with objective short sleep duration have a blunted response to cognitive behavioral therapy for insomnia. Sleep 40:Zsw12

7. Benjamins JS, Migliorati F, Dekker K, Wassing R, Moens S, Blanken TF, Te Lindert BH, Mook JS, van Someren EJ (2017) Insomnia heterogeneity: characteristics to consider for data-driven multivariate subtyping. Sleep Med Rev 36:71-81

8. Blanken TF, Benjamins JS, Borsboom D, Vermunt JK, Paquola C, Ramautar J, Dekker K, Stoffers D, Wassing R, Wei Y, Van Someren EJW (2019) Insomnia disorder subtypes derived from life history and traits of affect and personality. Lancet Psychiatry 6(2):151-163

9. Budhiraja R, Roth T, Hudgel DW, Budhiraja P, DrakeCL (2011) Prevalenceand polysomnographic correlates of insomnia comorbid with medical disorders. Sleep 34:859-867

10. Crönlein T, Wetter TC, Rupprecht R, Spiegelhalder K (2020) Cognitive behavioral treatment for insomnia is equally effective in insomnia patients with objective short and normal sleep duration. Sleep Med 66:271-275

11. Edinger JD, Wyatt JK, Stepanski EJ, Olsen MK, Stechuchak KM, Carney CE, Chiang A, Crisostomo MI, Lineberger MD, Means MK, Radtke RA, Wohlgemuth WK, Krystal AD (2011) Testing the reliability and validity of DSM-IV-TR and ICSD-2 insomnia diagnoses. Results of a multitrait-multimethod analysis. Arch Gen Psychiatry 68:992-1002

12. Ellis JG, Gehrman P, Espie CA, Riemann D, Perlis ML (2012) Acute insomnia: current conceptualizations and future directions. Sleep Med Rev 16:5-14

13. Ellis JG, Cushing T, Germain A (2015) Treating acute insomnia: a randomized controlled trial of a "single-shot" of cognitive behavioral therapy for insomnia. Sleep 38:971-978

14. Galbiati A, Sforza A, Leitner C, Castelnovo A, D'Este G, Ferini-Strambi L, Manconi M, Castronovo V (2021) The reliability of objective total sleep time in predicting the effectiveness of cognitive-behavioral therapy for insomnia. Sleep Med 82:43-46

15. Kleitman N (1963) Sleep and wakefulness (revised edition). University of Chicago Press, Chicago

16. Lack LC, Gradisar M, van Someren EJ, Wright HR, Lushington $\mathrm{K}$ (2008) The relationship between insomnia and body temperatures. Sleep Med Rev 12:307-317

17. Lovato N, Micic G, Lack L (2021) Sleep misestimation among older adults suffering from insomnia with short and normal objective sleep duration and the effects of cognitive behavior therapy. Sleep 44:zsaa250 
Tipps rund ums Publizieren

18. McCrae CS, Lichstein KL (2001) Secondary insomnia: diagnostic challenges and intervention opportunities. Sleep Med Rev 5:47-61

19. Miller CB, Espie CA, Bartlett DJ, Marshall NS, Gordon CJ, Grunstein RR (2018) Acceptability, tolerability, and potential efficacy of cognitive behavioural therapy for Insomnia Disorder subtypes defined by polysomnography: a retrospective cohort study. Sci Rep 8:6664

20. Morin $C M$, Drake $C L$, Harvey AG, Krystal AD, Manber R, Riemann D, Spiegelhalder K (2015) Insomnia disorder. Nat Rev Dis Primers 1:15026

21. Ohayon MM (2002) Epidemiology of insomnia: what we know and what we still need to learn. Sleep Med Rev 6:97-111

22. Pillai V, Roth T, Drake CL (2015) The nature of stable insomnia phenotypes. Sleep 38:127-138

23. Qaseem A, Kansagara D, Forciea MA, Cooke M, Denberg TD (2016) Management of chronic insomnia disorder in adults: a clinical practice guideline from the American College of Physicians. Ann Intern Med 165:125-133

24. Riemann D, Baum E, Cohrs S, Crönlein T, Hajak G, Hertenstein E, Klose P, Langhorst J, Mayer G, Nissen C, Pollmächer T, Rabstein S, Schlarb A, Sitter H, Weeß HG, Wetter T, Spiegelhalder K (2017) S3Leitlinie Nicht erholsamer Schlaf/Schlafstörungen - Kapitel Insomnie bei Erwachsenen, Update 2016. Somnologie 21:2-44

25. Riemann D, Baglioni C, Bassetti C, Bjorvatn B, Dolenc Groselj L, Ellis JG, Espie CA, Garcia-Borreguero D, Gjerstad M, Goncalves M, Hertenstein E, Jansson-Frojmark M, Jennum PJ, Leger D, Nissen C, Parrino L, Paunio T, Pevernagie D, Verbraecken J, Weess H-G, Wichniak A, Zavalko I, Arnardottir ES, Deleanu O-C, Strazisar B, Zoetmulder M, Spiegelhalder K (2017) European guideline for the diagnosis and treatment of insomnia. J Sleep Res 26:675-700

26. Schlack R, Hapke U, Maske U, Busch M, Cohrs S (2013) Frequency and distribution of sleep problems and insomnia in the adult population in Germany: results of the German health interview and examination survey for adults (DEGS1). Bundesgesundheitsblatt Gesundheitsforschung Gesundheitsschutz 56(5-6):740-748

27. Vgontzas AN, Fernandez-Mendoza J, Liao D, Bixler EO (2013) Insomnia with objective short sleep duration: the most biologically severe phenotype of the disorder. Sleep Med Rev 17:241-254

28. Vgontzas AN, Puzino K, Fernandez-Mendoza J, Krishnamurthy VB, Basta M, Bixler EO (2020) Effects of trazodone versus cognitive behavioral therapy in the insomnia with short sleep duration phenotype: a preliminary study. J Clin Sleep Med 16:2009-2019

29. Walsh JK, Coulouvrat C, Hajak G, Lakoma MD, Petukhova M, Roth T, Sampson NA, Shahly V, Shillington A, Stephenson JJ, Kessler RC (2011) Nighttime insomnia symptoms and perceived health in the America Insomnia Survey (AIS). Sleep 34:997-1011

\section{Das Wichtigste in Kürze: Literaturangaben}

Bitte achten Sie darauf, dass das Literaturverzeichnis alle im Text zitierten Literaturangaben enthält und Literaturangaben, die im Literaturverzeichnis stehen, mindestens einmal im Text erwähnt werden.

Die Referenzen im Literaturverzeichnis sollten mit allen notwendigen Angaben nach den folgenden Beispielen aufgebaut sein:

- Beispiel Zeitschrift: Nordmeyer SD, Kaiser G, Schaper A et al. (2022) Das Dreisäulenmodell der klinischen Toxikologie. Monatsschr Kinderheilkd 170, 613-620.

https://doi.org/10.1007/s00112-02201520-w

- Beispiel Buch: Zernikow B, Printz M (2022) Praktische Schmerztherapie. In: Zernikow, B. (eds) Pädiatrische Palliativversorgung-Schmerzbehandlung und Symptomkontrolle. Springer, Berlin, Heidelberg.

https://doi.org/10.1007/978-3-662-

63148-5 2

- Beispiel Online: http://www.springermedizin.de. Zugegriffen: 01. März 2022 